\title{
A COMPARATIVE STUDY OF THE CHANGE IN TOWNSCAPE IN HISTORICAL BLOCKS OF XI'AN, CHINA, AND KYOTO, JAPAN
}

\author{
*Kun Yuan ${ }^{1}$, Hirokazu Abe ${ }^{2}$, Kensuke Yasufukü and Akira Takahashi ${ }^{2}$ \\ ${ }^{1}$ Dept. of Global Architecture, Osaka University, Japan; ${ }^{2}$ Cybermedia Center, Osaka University, Japan \\ *Corresponding Author, Received: 06 Oct. 2021, Revised: 01 Dec. 2021, Accepted: 09 Jan. 2022
}

\begin{abstract}
This paper uses space syntax to analyze discrepancies in the urban spatial structures of two historical cities during the modernization process. Axial line maps of the main urban areas of Xi'an, China, and Kyoto, Japan, are compared from the beginning of their modernization periods to the present day. Changes in the cities' topological centers are analyzed, and the global and local relationships in their main urban areas are explored. The primary outcomes of the study are as follows: 1) With the expansion, the topological centers of the main two urban areas have changed. From (1902-2020), Kyoto's topological centers expanded simultaneously horizontally and vertically. Similarly, Xian's center expanded from one (bell tower) in 1949 to two in 2019. For both cities, Global Integration Value (Int. V global) grew, notably in Kyoto, during the modernization era. Long-distance accessibility also increased, showing the two cities' drive toward motorization. 2) Average Int. V global change rates for the local historical blocks of Old Xi'an city were different across local and global growth rates of urban space, emphasizing the local development feature of the block evolution. In Kyoto's three wards, Int. V global change rates were close to the global change rate, showing a consistent change in Kyoto's global integration with a higher consistency in global street construction. 3) The correlation of global integration and local integration, as represented by intelligibility (R2), increased in both Xi'an and Kyoto, indicating that the solidarity of the respective regions increased during the modernization process. This is especially true for Kyoto.
\end{abstract}

Keywords: Capital Cities, Morphological Change, Space Syntax, Xi'an, Kyoto

\section{INTRODUCTION}

Xi'an, China, and Kyoto, Japan, are ancient cultural centers whose main urban areas have developed around a chessboard network of roads. In terms of their morphology, the two have similarities, but they also have had distinct development features throughout their modernization.

The Ming City District is the core area of Xi'an, which was built based on the imperial city of the Sui and Tang Dynasties during the Hongwu Period of the Ming Dynasty, and which has retained many of its original areas [1]. The Ming City District is the center of Xi'an's urban human settlements, especially in its southwest area's remaining historic and cultural blocks of Beiyuan Gate and Sanxue Street, which inherited the best historical characteristics of the region and streets. With the founding of the People's Republic of China (1949), China began its modernization construction. [2] Since that time, Xi'an has experienced four master plans, and its urban areas have expanded dramatically to the East, South, and West from the old city, growing from 13.2 to 724 square kilometers; during this period, the population has also increased, from 2.27 to 10.2 million [1]. With the rapid expansion and development of the city area, the form and structure of the main urban area within the city wall has changed significantly, and a large number of historical alleys have been rebuilt while others have disappeared in the historic blocks of the main city.

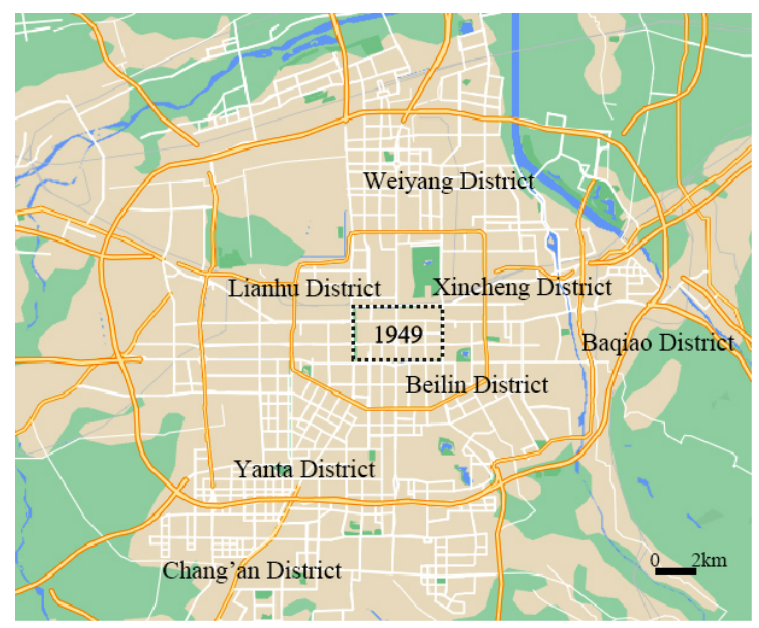

Fig.1 Map of Xi'an ( From 1949 to 2019)

Japan began its modernization construction in the Meiji period (1868-1912), during which time the new Japanese government began to restore Kyoto [3]. Several urban planning measures were carried out, widening streets and creating a ring road, all of which had the effect of accelerating the city's modernization. In the Showa period (1926-1989), 
the city merged with surrounding towns, rapidly and significantly expanding its urban area from 31 to 210 square kilometers. The population increased from 0.28 million in the Meiji period to 1.41 million [4,5]. The city's urban development is still mainly characterized by grid planning, and the main urban area is dominated by Kamigyo Ward, Nakagyo Ward, and Shimogyo Ward. As in Xi'an, the modernization construction in Kyoto has greatly changed the urban area and street morphology of the city through urban planning.

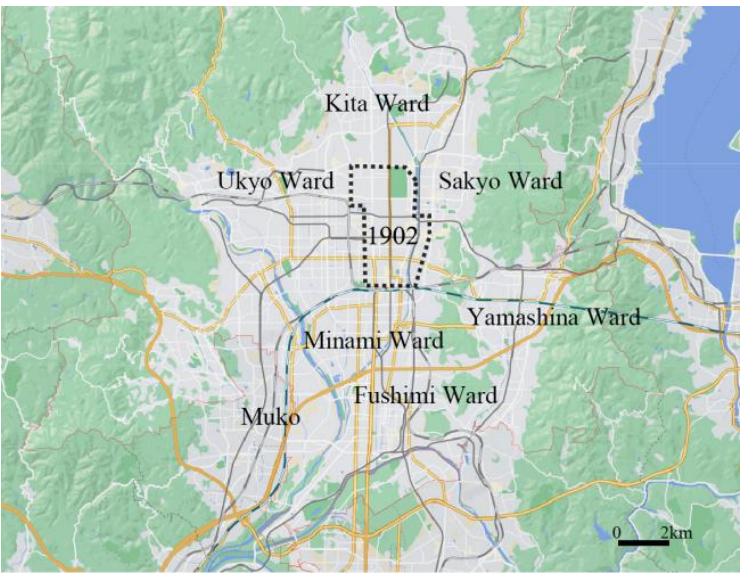

Fig.2 Map of Kyoto ( from 1902 to 2020)

Previous studies of the urban morphology of the two cities include a study by Onishi [2], who conducted a detailed investigation of the changes in the townscape of Xi'an's historic center and compared them to developments in Kyoto. Kang [6] studied the spatial morphology characteristics of Xi'an's Historic District using traditional spatial morphology analysis, mostly in terms of spatial layout, texture, street scale, and plane form. Compared to investigative analyses that focus on traditional morphological characteristics, more recent spatial morphology analysis is mainly carried out using quantitative methods and is mainly focused on space syntax with a GIS data platform. In an empirical and quantitative study of large-scale urban streets and roads in three separate periods, Huang [7] investigated the complex evolution of the morphological characteristics of the typical urban Hui culture from the morphological middle, street structures, and network systems perspective, and examined the shift in the topological relationship between historic block space and urban whole space; however, there are few comparative studies between similar blocks.

Space syntax is important for extracting the topological structures of a city, particularly when comparing two or more cities. Kigawa [3] conducted an analysis of Kyoto's spatial configurations throughout the ages and its urban planning project based on space syntax, revealing that streets had a strong impact on Kyoto's transformation from its early modern to modern form. Kigawa [8] also used space syntax to analyze the process by which the castle cities had been reformed. In studying the process of urban modernization in several of the castle cities of Japan, he found distinct differences among the cities. In China, a comparative study of urban space based on space syntax was conducted by Wang et al. [9], who selected cities with wide differences in road networks and land configurations. Analyzing the axial line, the study explored differences and commonalities. Such studies help us to understand core area aggregation and urban morphology evolution inertia. However, comparative studies involving cities in two different countries that have a similar space structure initially but which later experience different development patterns are rare.

This paper compares the urban spatial structure changes in Xi'an and Kyoto in their respective modern city construction. In the process, we not only make a horizontal comparison of similar cities in different development stages but also provide a vertical analysis of the relationship between their global and local aspects. Using a comprehensive quantitative analysis of the current urban development situation and its distinctive characteristics, we compare axis maps of the main urban areas of Xi'an and Kyoto in different years, and analyze the changes in their topological centers as well as the global and local relationship in their main urban areas.

\section{METHOD}

Space syntax is a set of theories and analytical tools introduced in "The Social Logic of Space" by Hillier and Hanson in 1984 [10]. The topological analysis focuses on the connection between spaces.

We examined the street network of the historical capital districts in two steps:

First, we use an axial analysis to investigate the street network, then compare the axis maps of Xi'an and Kyoto in different years, tracing the changes in their topological centers. (The axial map is constructed by taking an accurate map and drawing a set of intersecting lines through all the spaces of the urban grid so that the grid is covered and all rings of circulation are completed.)

Because the urban morphologies of the two cities have undergone dramatic changes in modern times, we chose the beginning period of the modernization construction of the two cities as the contrastive object. The starting point examined in this study for Xi'an is the founding of the new China in 1949; the starting point for Kyoto is the year of the earliest measured map (1902) in the Meiji period of the new Japanese government.

The study area for Xi'an focuses on the main 
urban areas that served as the Ming castle city (13.2 $\left.\mathrm{km}^{2}\right)$. The main study area for Kyoto $\left(21.2 \mathrm{~km}^{2}\right)$ consists of three districts (Kamigyo Ward, Nakagyo Ward, and Shimogyo Ward). Concerning Xi'an, the key objects used in the study include four maps of narrow streets in the old city 1949 road network, together with a remote sensing image of Xi'an in 2019. In the case of Kyoto, the key objects are a field survey map from 1902 and a cadastral map from 2020.

To analyze the configuration layout of each city, we translated the actual spatial structure into an axial map (Fig.3), which is the last set of longest lines drawn tangent to vertices that can see each other. For our topological analysis, the axial map was projected onto a justified graph in which the axial lines are represented as nodes, and the intersecting lines are the connections between the nodes [10].

Fig.3 shows a justified graph that has been converted from an axial map [11]. A justified graph is one in which a node is drawn at the base, and all the points of various depths are drawn above it. Specifically, all the points at depth 1 from the starting point are aligned horizontally immediately above it, all the points at depth 2 from that point are drawn above those at depth 1 , and so on, until all levels of depth from the initial point are accounted for. The topological total depth and mean depth can then be determined from the justified graph.

A depth value represents the convenience of moving from one space to another. The topological distance between two adjacent nodes is one step. The shortest topological distance between any two nodes-that is, the number of spatial transformations-is expressed as the depth value between the two nodes. The larger the value, the deeper the element is hidden in the network of the study area.

Topological total depth (TD) is the cumulative total of the fewest topological depth paths between all pairs of nodes. MD (mean depth) is the average of the minimum number of steps from one node to all the other nodes in the system, which eliminates the effect of numbers.

Next, integration is introduced as an important result of the axis analysis. Its value is derived from the value of the topological depth, as in Fig. 4. By analyzing the integration value, it is possible to determine the degree of a street and how topologically shallow or deep the entire system is from a point on the street.

An integration value essentially describes the "accessibility" of an element in the study area network. The higher the depth value, the lower is the degree of integration, that is, the worse is the connectivity and vice versa. More specifically, axial local integration is defined as the integration values of the axial lines at radius 3 (root plus two topological steps from the root) which can be used to

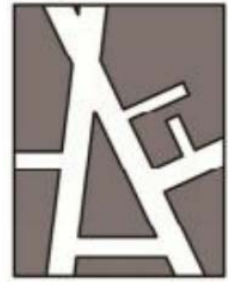

A.Street Map

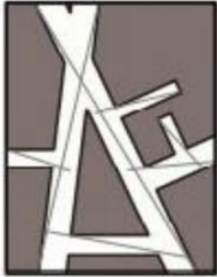

B.Axial Map

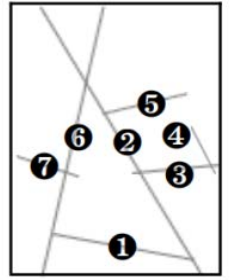

C.Axial line and Number

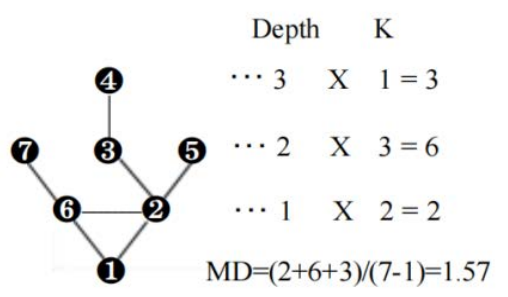

D.Justified Graph

Fig.3 Axial analysis

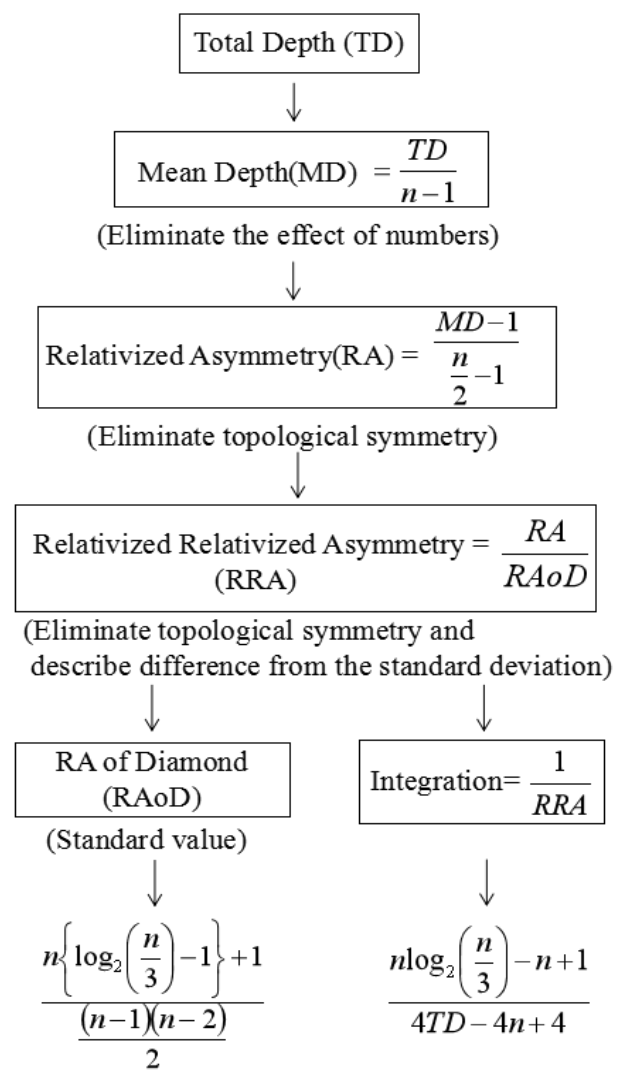

Fig.4 Flow chart from depth to integration $\mathrm{n}$ : total number of vertices

represent a localized picture of integration. Axial global integration is defined as the integration values of axial lines at an infinite radius which can be used to represent the integration pattern at the largest scale. 
Finally, the integration core can be used to represent the change and transfer of the topological center, consisting of the most integrating $10 \%$ of the lines $[10,12]$.

In the second step, the results of the topological analysis conducted in the previous step are used to analyze the global and local relations of the respective main urban areas.

Axial intelligibility indexes the degree to which the number of immediate connections that a line has is a reliable guide to the importance of that line in the system as a whole. (Specifically, it is the correlation between local and global elements). Thus, intelligibility is introduced as a way to describe the correlation between local integration and global integration and as the means to measure whether the local space and the global space environment are related and unified. This relationship indicates whether an area is intelligible in its global context $[13,14]$.

Intelligibility: $R^{2}=\frac{\left[\sum\left(I_{3}-\overline{I_{3}}\right)\left(I_{n}-\overline{I_{n}}\right)\right]^{2}}{\sum\left(I_{3}-\bar{I}_{3}\right)^{2} \sum\left(I_{n}-\bar{I}_{n}\right)^{2}}$

The value of intelligibility $\left(\mathrm{R}^{2}\right)$ is calculated by Equation 1, where $I_{n}$ is the global integration of any axis in space, and $I_{3}$ is the three-step integration value of any axis in space. $\bar{I}_{n}$ and $\bar{I}_{3}$ are the mean values of global integration and three-step integration, respectively.

For Eq. (1), if the intelligibility of the local space is high, the indication is that the centrality of local space can be integrated into the global spatial structure. The natural movement of local space can not only bring movement economy; it can also produce a greater multiplier effect in the process of functional interaction with the movement economy of global space. On the other hand, if the intelligibility of local space is low, the indication is that the local centrality of the regional urban space cannot be integrated with the global space and is thus relatively isolated [12,15].

\section{RESULTS AND DISCUSSION}

\subsection{Changes In The Main Urban Areas Of Xi'an And Kyoto}

\subsubsection{Main urban area of Xi'an}

The two axial maps of Xi'an (1949 and 2019) shown in Fig. 5, allow us to examine the change in the urban topological center. With the development of lattice sub-trunk road planning, many narrow alleys were expanded and new roads throughout the castle district were constructed. The global integration value (Int. V global) of Lianhu Road, East 5th Road, and West 5th Road to the north of the central axis, Jiefang Road, and Heping Road to the east of the Central Axis is high. This suggests that the topological center of the street network has extended to the north side roads of the main road centered on the bell tower.
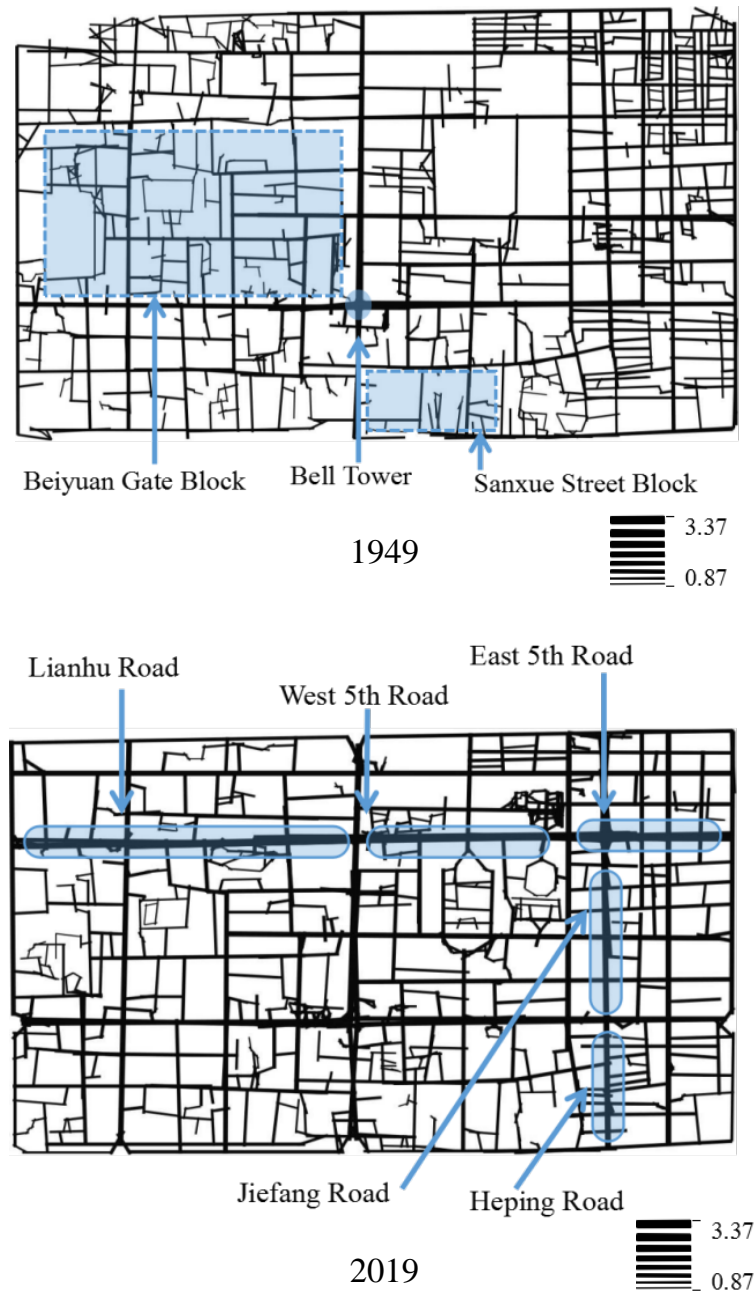

Fig.5 Axial maps with Global int.V for Xi'an in 1949 and 2019

The remaining historical and cultural blocks of Beiyuan Gate and Sanxue Street in the western and southern areas of Ming city, with low Int .V global values, were far from the topological center of the main crossroad centered on the bell tower and the northeastern part of the castle city in 1949. The average global integration values of the two historical blocks are 1.56 and 1.58, respectively, which is lower than the average global value of 1.71 in 1949. By 2019, the average global integration value had increased from 1.71 to 1.91 , over which time the values for the two historical blocks increased to 1.88 and 1.74 , respectively, both of which are lower than the 1.91 global average. However, the percentage change for the Beiyuan Gate block (18\%) is greater than the global average (12\%), while the percentage change for the Sanxue Street Block (10\%) is slightly lower. The indication is that the historical blocks in the main city area 
experienced a high degree of local change with urban global space development. The local development in the evolution of the historic blocks is clear.

\subsubsection{Main urban area of Kyoto}

Fig. 6 shows the 1902 and 2020 axial maps with Int. $\mathrm{V}$ global for Kyoto. The streets with high integration values based on the results of the axial analysis are more darkly shaded.
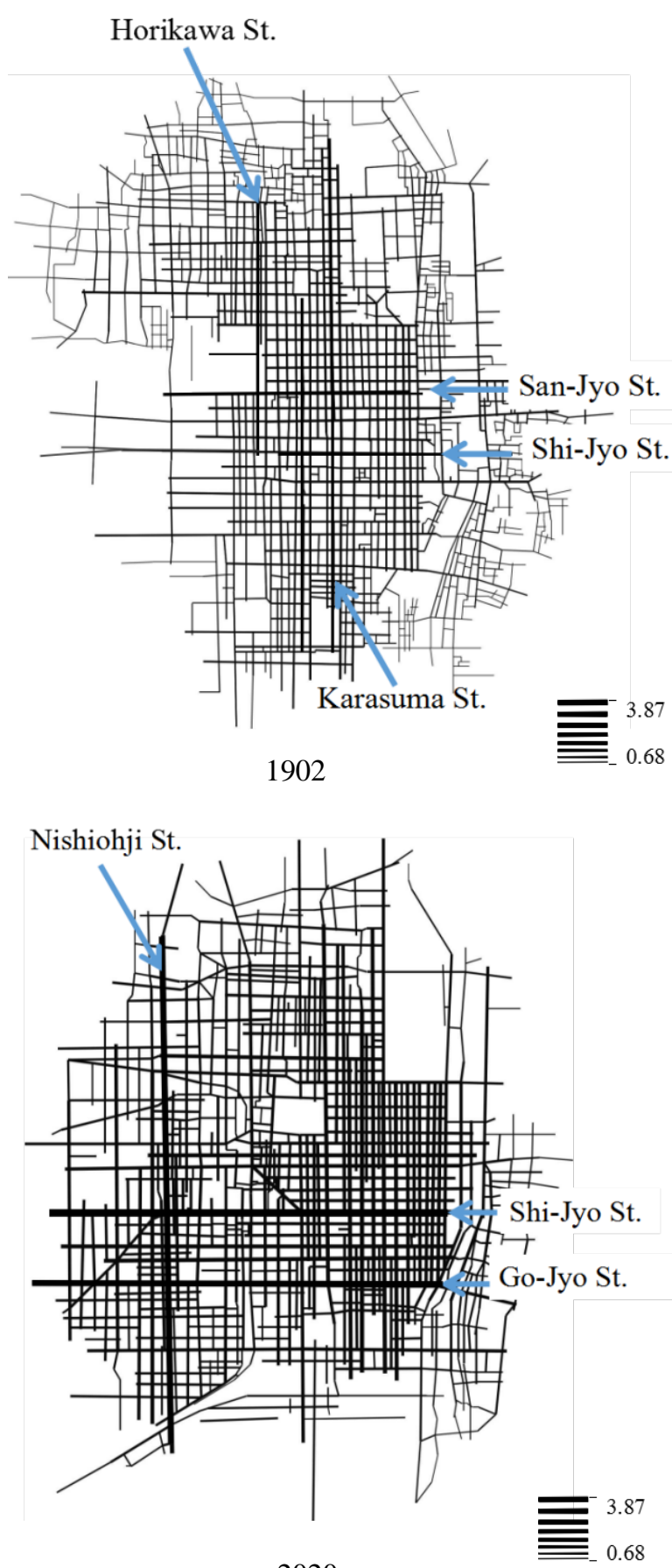

2020

Fig.6 Global integration values for Kyoto in 1902 and 2020
In 1902, the highest integration values are for Karasuma Street, Horikawa Street, and San-Jyo Street, which constitute the urban topological center, as seen in the two maps. The highest vertical axial global Int. V extended outward and westward to Nishiohji street in 2020. The highest horizontal axial global Int. V expanded from San-Jyo Street to shiJoy Street. High connectivity is also seen for Go-Jyo Street and Shichi-Jyo Street. The topological center extended outward in both the horizontal and vertical directions simultaneously. The average global integration values of the three Kyoto wards increased from 1.38, 1.62, and 1.54, respectively, in 1902 to 2.18, 2.46, and 2.37 in 2020, corresponding to respective change rates of $55.7 \%, 51.8 \%$, and $53.9 \%$. The fact that the change rates are not substantially different from the global change rate (53.6\%) indicates a strong consistency in global street construction.

As can be seen here, the topological centers of both cities experienced an outward expansion. In the main urban area of Kyoto, the consistency of street construction is stronger and the change in global accessibility is more obvious. In Xi'an, the global change in the main city is not nearly as clear. While Xi'an's main urban area has been strongly affected by the urban expansion outside of the castle city, the residential areas inside the city wall also have obvious characteristics of local development. The external driving force of urban global space development and the internal traction of block evolution appear to have interacted with each other to jointly promote the deepening development of the structure of the historic blocks.

\subsection{Scatter Diagram Analysis}

Scatter diagrams for Xi'an showing Int. V global on the horizontal axis and Int. $\mathrm{V}$ local on the vertical axis provided in Fig.7. The distribution shapes of the scatter points in 1949 and 2019 show little similarity. In 1949, Int. V global was relatively low, and the scatter points are concentrated in the lower-left portion of the diagram. In 2019, the concentration of the scatter points has shifted toward the middle and the average Int. V global has increased, indicating that the Int. V global of Xi'an's axial line became stronger in the process of the city's modern urban spatial development.

Fig.8 gives the Int. V (global and local) scatter diagrams for Kyoto. As shown, the center of the dense area of the scatter points moves rightward from 1902 to 2020. The average value of Int. V global increased markedly as the global integration value of most of the scatter points was greater than 1.0. Such results indicate that Int. V global grew substantially stronger and long-distance accessibility increased during the modernization period. 

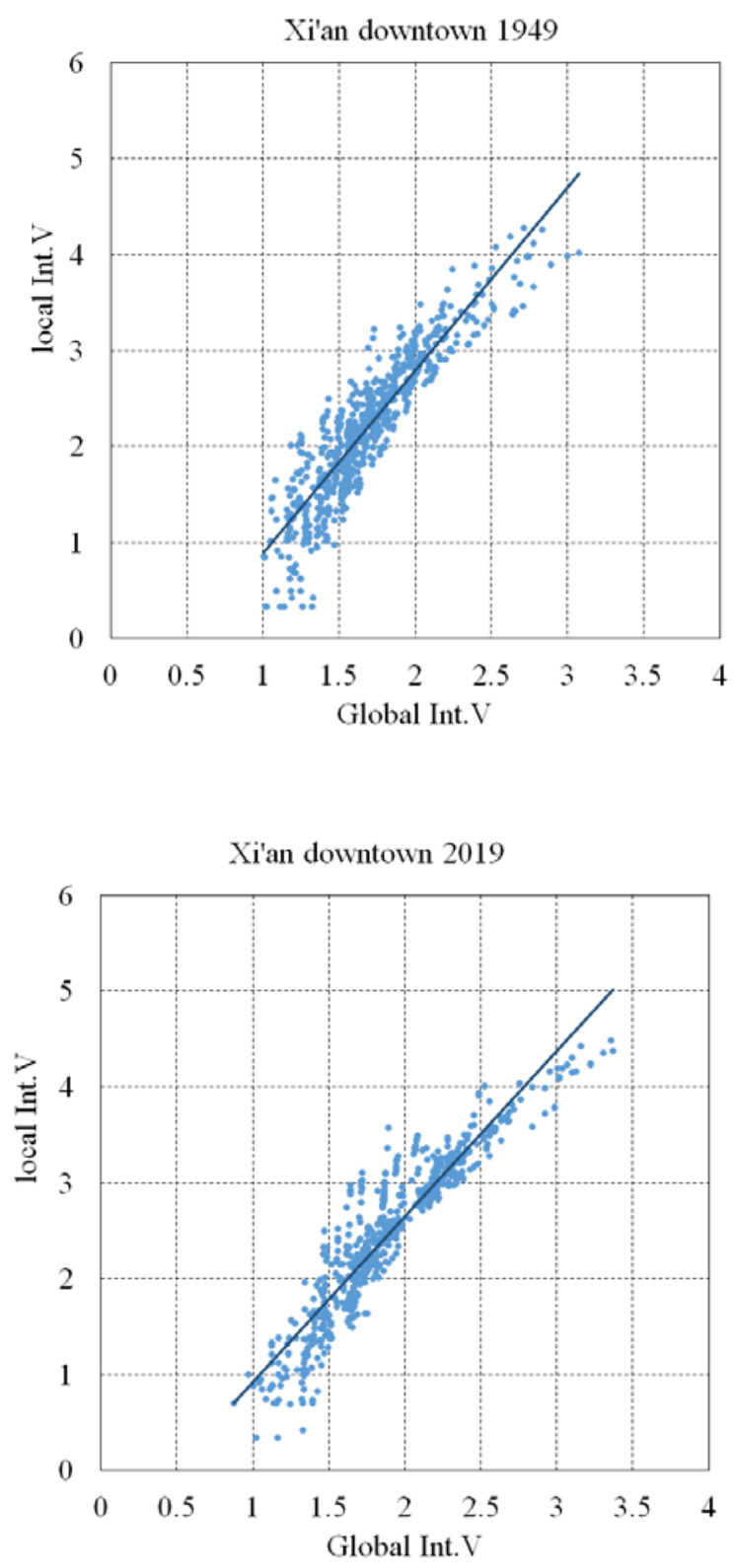

Fig.7 Int. V (global and local) scatter diagrams for Xi'an in 1949 and 2019

Table 1 gives key parameters of the scatter plots in Fig.7 and Fig.8. Of particular note is the slope of the regression line fitted to the Xi'an (Fig.7) scatter points, which decreases from 1.905 in 1949 to 1.725

Table 1 Parameter comparison of Fig.7 and Fig.8
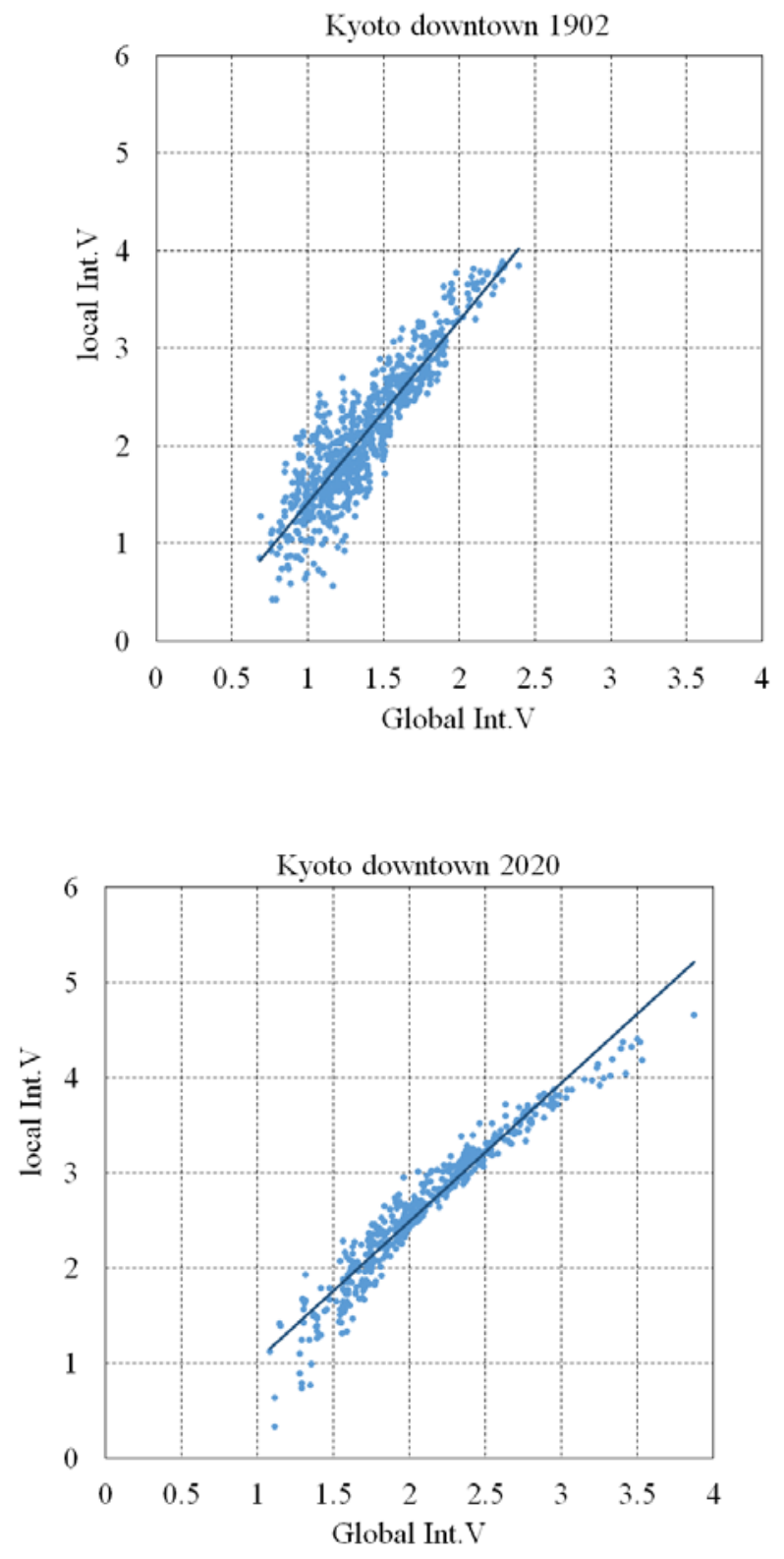

Fig.8 Int. V (global and local) scatter diagrams for Kyoto in 1902 and 2020

in 2019. The slope of the regression line for the Kyoto scatter diagrams (Fig.8) also decreases, from 1.868 in 1902 to 1.454 in 2020 . The fact that the slopes decrease means that the scatter points move rightward (i.e., Int. V global becomes stronger). In general, urban networks with a higher Int. V global are better suited for long-range accessibility, since

\begin{tabular}{ccccc}
\hline & 1902, Kyoto & 2020, Kyoto & 1949, Xi'an & 2019, Xi'an \\
\hline Regression Line & $\mathrm{y}=1.868 \mathrm{x}+0.4555$ & $\mathrm{y}=1.4549 \mathrm{x}+0.4241$ & $\mathrm{y}=1.9052 \mathrm{x}+1.0203$ & $\mathrm{y}=1.7255 \mathrm{x}+0.803$ \\
R2(Intelligibility) & 0.8054 & 0.9319 & 0.8229 & 0.8509 \\
Global Int V(mean) & 1.3774 & 2.1162 & 1.711 & 1.908 \\
Local Int V(mean) & 2.1174 & 2.6564 & 2.2396 & 2.4893 \\
\hline
\end{tabular}


Int. V global is calculated with up to all topological depths in Depthmap and is closely related to motor vehicle traffic. In contrast, urban networks with a higher Int. V local is better suited for short-range accessibility related to pedestrian traffic. From the basics of these, it can be inferred that the modernization of the two cities was heading toward motorization.

The intelligibility $\left(\mathrm{R}^{2}\right)$ value for Xi'an increased from 0.8229 (1949) to 0.8509 (2019), while the value for Kyoto increased from 0.8054 (1902) to 0.9319 (2020). Hillier suggests that intelligibility is used to describe the correlation between local integration and global integration and

measures whether the local space and the global space environment are related and unified. This relationship presents if an area is intelligible in its global context [13].

Hillier also suggests that if the intelligibility value of local space is low, the implication is that the local centrality of the regional urban space cannot be integrated with the global space and is thus relatively isolated [15]. The value of intelligibility $\left(\mathrm{R}^{2}\right)$ increases in both Xi'an and Kyoto, meaning that the solidarity of the respective regions has increased during the modernization process.

\section{CONCLUSIONS}

This paper sought to uncover similarities and differences in the evolution of the historical cities of Xi'an and Kyoto by using space syntax. Through axis analysis of the two main urban areas in different years, the topological centers and the global and local relations of the main urban areas were compared. The analysis produced three important findings:

1) With the expansion of the urban area, the topological centers changed correspondingly. The topological center in the main urban area of Kyoto expanded outward from 1902 to 2020 in both the horizontal and vertical directions simultaneously. In Xi'an, the area expanded from one topological center centered on the bell tower in 1949 to two topological centers in 2019. Int. V global increased markedly for the two cities (especially for Kyoto), and long-distance accessibility increased during the period of modernization. The implication is that the modernization of the two cities was heading toward motorization.

2) The average Int. $V$ global change rates of the local historical blocks in the old Xi'an city show a difference in the local change and the global growth of its urban space and make evident the local development feature of block evolution. On the other hand, the Int. $\mathrm{V}$ global change rates for the three wards in Kyoto are similar to the global change rate, indicating a consistent change in global integration and strong consistency of global street construction.

3) The global and local integration correlation of the two major urban areas is reinforced by the characteristic curve and index analysis, with the two exhibiting strong similarities. The intelligibility $\left(\mathrm{R}^{2}\right)$ value increases in both Xi'an and Kyoto, meaning that the solidarity of the respective regions has increased during the modernization process. In this regard, Kyoto is stronger than Xi'an.

\section{REFERENCES}

[1] Xi'an Municipal Bureau of Statistics, Xi'an Statistical Yearbook, China Statistics Press, 2020.

[2] Ohnishi, K., A Study on the Change of Townscape in the Historic Center in Xi'an City, China, Journal of the City Planning Institute of Japan, Vol. 28, 1993.

[3] Kigawa, T. and Furuyama, M., Study on a Vector in Kyoto's Modernization using Space Syntax, Journal of the City Planning Institute of Japan, Vol. 40-3, 2005, pp. 139-144.

[4] Ohnishi, K., The Beauty of Kyoto-The Theory of Conservation and Regeneration, Kajima Institute Publishing Co., Ltd., 1992.05

[5] Kyoto Population, Japan Geographic Data Center, 2020.

[6] Yitong, K., Sunwei, L. and Wei, F., The Beilin Historic District, Xi'an Spatial Morphology Characteristics Analysis, Journal of Architecture and Culture, Vol. 9, 2017.

[7] Jiaying, H., Study on the Construction and Morphology Transition of the Drum-tower Hui Community in Xi'an, Doctoral Dissertation of South China University of Technology, 2010.

[8] Kigawa, T. and Seo, K. W., Using Space Syntax to trace the Japanese urban renovation from JYOKAMACHI to modern cities What formed the city, the Centre or the Boundary?, Proceedings of the 11th International Space Syntax Symposium, 2009.

[9] Jiejing, W. Fang, W. and Rui, L., Space Syntax Based Urban Morphology Comparison, Journal of Planners, Vol. 28, 2012.6.

[10]Hillier, B. and Hanson, J., The Social Logic of Space, Cambridge University Press: Cambridge, 1984.

[11] Woochul N. A. and Abe H., A Morphological Examination of Street Networks and Building Condition in the Old Castle District of Daegu, Korea, 15th international conference on geometry and graphics, 2012.

[12] Hillier, B., Hanson, J., Grajewski, T., and Xu, J., Natural movement: or, configuration and attraction in urban pedestrian movement., Environment and Planning B: Planning and Design, Vol. 20, 1993, pp. 29-66.

[13] Hillier, B., Burdett, R., Peponis, J. and Penn, A., 
Creating Life: Or, Does Architecture Determine Anything? Journal of Architecture and Behaviour, Vol. 3-3, 1987, pp. 237.

[14] Tuncer, E., Perception, and Intelligibility in the Context of Spatial Syntax and Spatial Cognition., Proceedings of the 6th International Space Syntax Symposium, 2007.
[15] Hillier, B., Space is the Machine, Cambridge University Press: Cambridge, 1996, pp. 149-200.

Copyright () Int. J. of GEOMATE All rights reserved, including making copies unless permission is obtained from the copyright proprietors. 